\title{
THE SPHENOIDAL SINUS IN OPTIC NERVE DECOMPRESSION
}

\author{
P. D. GORMLEY, J. BOWYER, N. S. JONES and R. N. DOWNES \\ Nottingham
}

\begin{abstract}
SUMMARY
Surgical decompression of the orbit may be necessary in dysthyroid patients with compressive optic neuropathy. Two-wall decompression with a trans-antral or a trans-conjunctival approach is commonly used. However, in some patients the initial improvement following this surgical procedure is not maintained. Although this may be due to disease progression, a variant of orbital anatomy can contribute to sub-optimal decompression. We report three cases in which recurrence of compressive optic neuropathy occurred following two-wall decompression. The sphenoidal sinus was placed anteriorly in these patients. Further decompression which included the lateral wall of the sphenoidal sinus resulted in improvement. An endoscopic approach provides superior access and visibility for decompression of the optic nerve into the sphenoidal sinus, and this approach may be the surgical treatment of choice in these cases.
\end{abstract}

The incidence of patients with dysthyroid eye disease who develop compressive optic neuropathy is in the order of $8.6 \%$. $^{1}$ These patients are usually treated in a stepwise manner ${ }^{2}$ beginning with systemic steroid therapy. ${ }^{3}$ In some patients medical treatment fails and surgical intervention is required. The key area of surgical decompression is the orbital apex and many authors have described various surgical techniques to decompress this area. The importance of posterior dissection and the removal of apical bone has been stressed. ${ }^{4.5}$ In this area the anatomical variability of the sphenoidal sinus and the relationship of the carotid artery and the optic canal are extremely important. ${ }^{6}$ We describe 3 patients with dysthyroid eye disease and compressive optic neuropathy in whom anterior placement of the sphenoidal sinus occurred as a variant of the normal orbital anatomy.

\footnotetext{
From: Departments of Ophthalmology and Otolaryngology, Queens Medical Centre, Nottingham, UK.

Correspondence to: $\mathrm{Mr} \mathrm{R}$. N. Downes, Queens Medical Centre, Nottingham NG7 2UH, UK. Tel: +44 (0115) 9249924. Fax: +44 (0115) 9709749 .
}

In patients with this orbital anatomy endonasal endoscopic decompression may be the best surgical approach.

\section{Case 1}

\section{CASE REPORTS}

A 31-year-old woman with a history of dysthyroid eye disease and insulin-dependent diabetes was admitted to our ward with symptoms and signs of bilateral optic nerve compression. She had had a left two-wall orbital decompression performed 4 months previously and was on systemic steroid and cyclosporin. On examination the visual acuities were $6 / 24$ right and 6/36 left corrected. Bilateral Holmes-Adie pupil precluded accurate assessment of relative afferent pupil reflex. Colour vision was impaired in both eyes (Ishihara plates). Hertel measurements were 23 and $24 \mathrm{~mm}$ on the right and left respectively. A full orthoptic assessment showed a small-angle left divergent strabismus and marked restriction of the extraocular movements in each eye in up-gaze, adduction and abduction.

A computed tomographic (CT) scan of the orbits demonstrated enlargement of the extraocular muscles with compression of both optic nerves posteriorly at the orbital apices. It was decided to perform a right and then a left two-wall orbital decompression, 3 days apart with a standard external approach. Visual acuities improved to $6 / 9$ bilaterally corrected following this surgery. No significant change in eye position or motility was noted. Systemic steroid and cyclosporin therapy was gradually tapered.

Two months after the operation the patient had a deterioration in the left visual acuity which did not respond to an increase in steroid and cyclosporin therapy. Two months later visual acuities were $6 / 9$ right and 6/24 left. Hertel measurements were 23 and $24 \mathrm{~mm}$ on the right and left respectively. Orthoptic assessment was similar to before surgery. A repeat CT scan showed a good result from the previous

Eye (1997) 11, 723-726 c) 1997 Royal College of Ophthalmologists 
decompression with herniation of orbital contents anteromedially into the ethmoidal sinus and inferiorly into the maxillary sinuses on both sides. However, the optic nerve had not been decompressed posteriorly at the orbital apex due to the anterior sphenoidal air cells (Fig. 1).

It was reasoned that a left endoscopic sphenoethmoidectomy might provide a better posterior orbital decompression compared with repeat surgery using a standard approach. An endoscopic decompression of the lateral wall of the sphenoid was performed. The sphenoid was opened, the lamina papyracea was excised and bone was drilled on the lateral aspect of the sphenoid using a sheathed Storz drill.

The post-operative course was uncomplicated. The patient was discharged from hospital the following day and the course of steroid and cyclosporin was gradually reduced. Three months later, the corrected visual acuities were 6/9 bilaterally. Hertel measurements were unchanged and no deterioration in ocular motility was noted. Medication had been tapered then discontinued. A repeat CT scan showed a good surgical result with bone removal from the medial wall of the sphenoidal sinus.

\section{Case 2}

A 40-year-old woman with dysthyroid eye disease and insulin-dependent diabetes presented in October 1994 with symptoms and signs of left optic nerve compression which had not responded to systemic steroid therapy. On examination, the visual acuities were $6 / 6$ bilaterally corrected. Right colour vision was full (Ishihara plates). Left colour vision was impaired. A left relative afferent pupillary defect was noted and there was $5 \mathrm{~mm}$ of left proptosis (Hertel

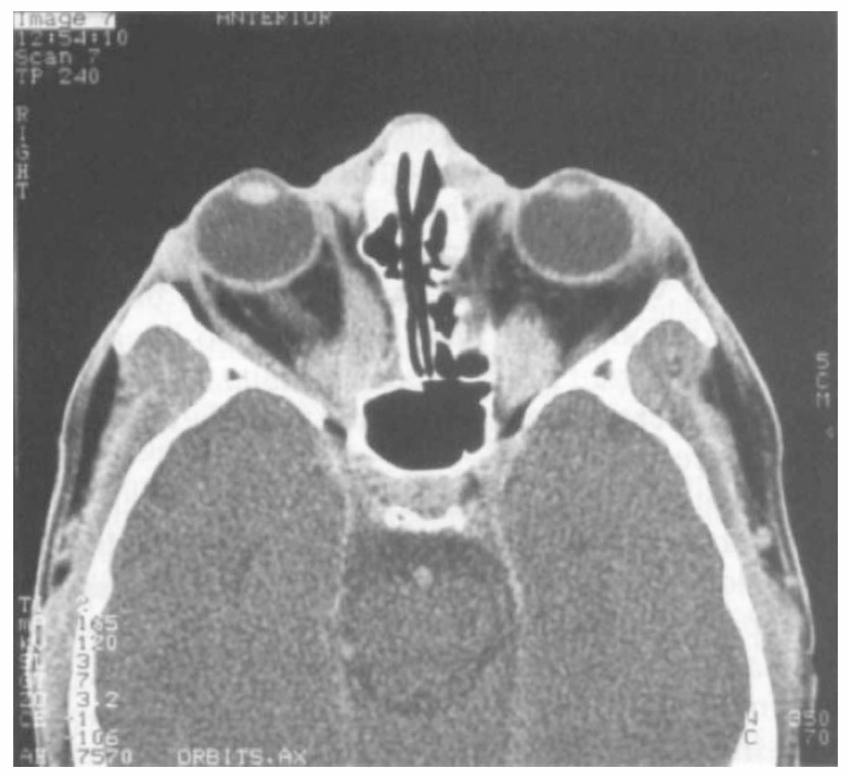

Fig. 1. Case 1. CT scan (axial plane) to show anterior placement of the sphenoidal air cells. measurements 20 and $25 \mathrm{~mm}$ on the right and left respectively). Extraocular movements were full in the right but markedly reduced in the left eye. CT scan showed moderately severe dysthyroid eye disease more pronounced on the left side with compression of the left optic nerve at the apex.

An external two-wall left orbital decompression was performed. Post-operatively, visual acuity remained stable, colour vision improved and the afferent pupillary defect resolved. Proptosis reduced to $3 \mathrm{~mm}$ and orthoptic assessment showed better movement in the left eye. Systemic steroid therapy was tapered. However, in December 1994 signs of recurrent left optic nerve compression were noted with deterioration in the clinical picture: although the left visual acuity was maintained at $6 / 6$, colour vision was impaired and there was a left afferent pupillary defect. Repeat CT scan showed prolapse of the medial rectus muscle as well as intraorbital fat into the ethmoidal sinus and soft tissue prolapse into the maxillary sinus, in keeping with previous surgery. Anterior placement of the sphenoidal sinus was noted.

A trial of systemic steroids failed to improve the clinical signs and it was decided to proceed to left endoscopic sphenoidal decompression under general anaesthetic 4 months later. The sphenoid was opened, the lamina papyracea was excised and bone was drilled and excised on the lateral aspect of the sphenoid to decompress the left optic nerve.

The immediate post-operative period was uneventful. The patient was discharged from hospital the following day on a gradually reducing dose of steroid therapy. At the time of last review, 9 months

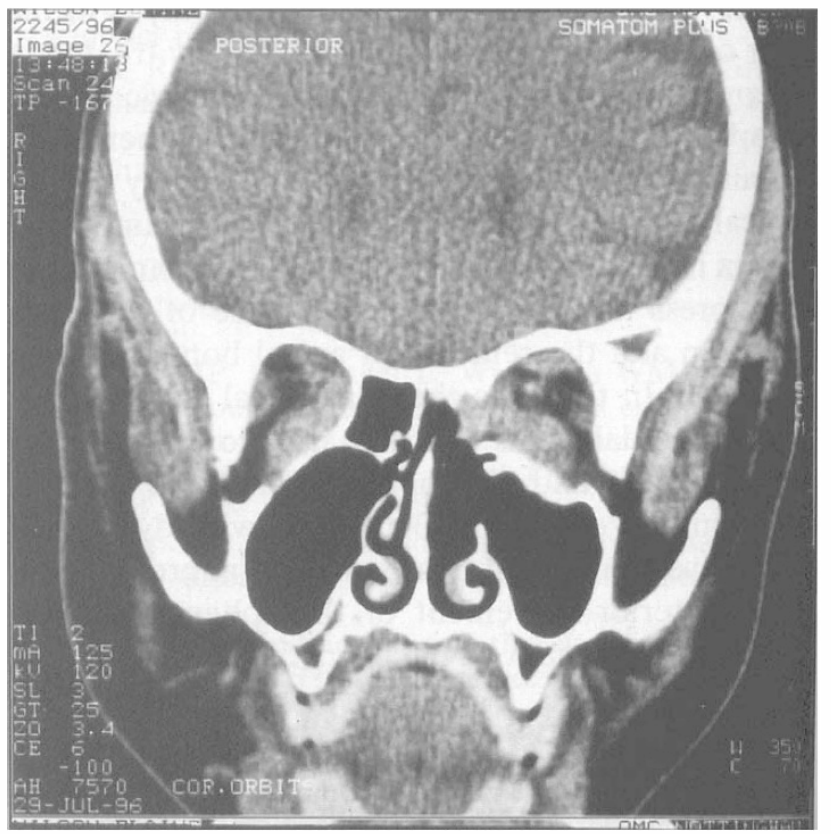

Fig. 2. Case 2. CT scan (coronal plane) to show decompression into the left sphenoidal sinus. 
later, visual acuities were $6 / 6$ bilaterally unaided. Colour vision was full in both eyes. There was no relative afferent pupillary defect and $1 \mathrm{~mm}$ of left proptosis only. No diplopia or motility problem had occurred. The patient had been off systemic steroid for 6 months. The post-operative CT scan shows excellent decompression into the left ethmoidal and maxillary sinus and marked decompression of the optic nerve posteriorly at the orbital apex into the sphenoidal sinus (Fig. 2).

\section{Case 3}

A 53-year-old woman with a history of dysthyroid eye disease presented with bilateral visual deterioration due to optic nerve compression. Right visual acuity was $6 / 24$ and the left $6 / 36$ corrected. A right three-wall orbital decompression and then similar left decompression 3 months later led to a slight initial improvement in the visual acuities but this was not maintained. Four months later the patient's visual acuities deteriorated to $6 / 60$ bilaterally corrected. A conservative approach to the management of the ophthalmic condition was adopted at this stage due to difficulty in controlling her systemic thyrotoxicosis.

Nine months later, the visual acuities were $6 / 60$ bilaterally corrected. Further medial wall orbital decompression was performed on the right orbit and on the left orbit 1 month later. However, visual acuities failed to improve significantly despite systemic steroid therapy (best recorded 6/24 bilaterally corrected, but not maintained). In addition, persistent post-operative diplopia required squint surgery. A right inferior rectus recession and then a left inferior oblique recession were performed with good result.

A repeat CT scan 22 months later showed the presence of residual posteromedial wall and anterior placement of the sphenoid bone on both sides. It was decided to proceed with further surgery. A right posteromedial wall orbital decompression with an external medial approach was performed to allow decompression into the anteriorly placed sphenoidal sinus. A similar procedure was performed to the left orbit 4 months later. However, there was no significant improvement in visual acuities after this surgery. Recurrence of diplopia required further squint surgery. A right medial and inferior rectus and a left medial rectus recession, positioned with adjustable sutures, 8 months later resulted in significant improvement in the patient's symptoms.

The visual acuities at time of last review 2 years after optic nerve decompression were $6 / 24$ bilaterally corrected. The post-operative CT scan (Fig. 3) confirmed a good surgical result with optic nerve decompression at each orbital apex. It was felt that the poor visual outcome was due to chronic optic

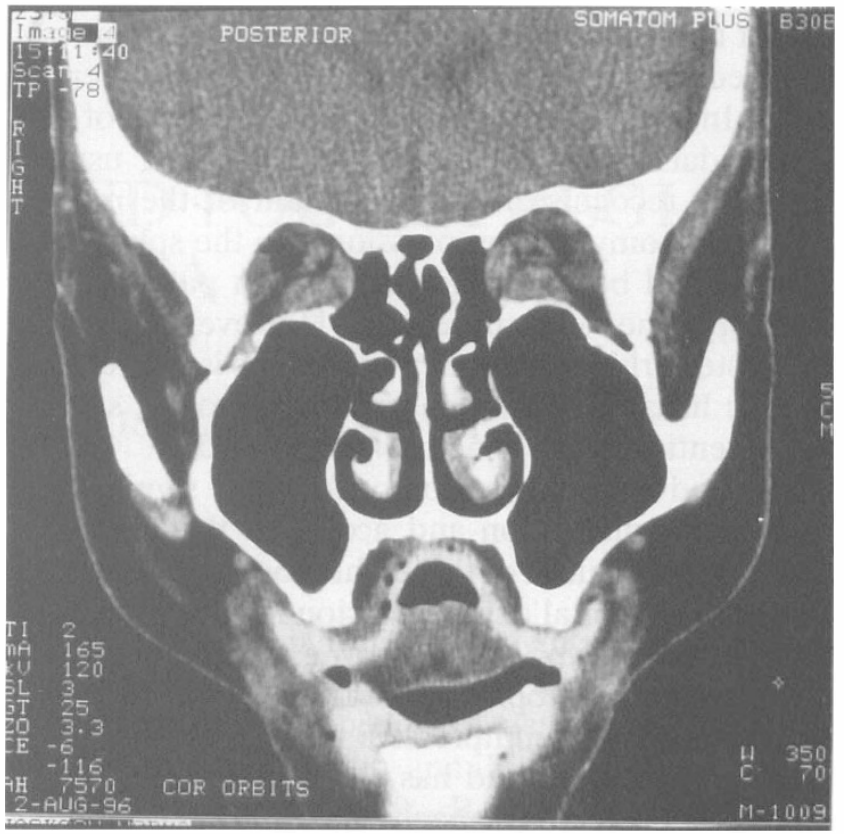

Fig. 3. Case 3. CT scan (coronal plane) to show bilateral surgical decompression.

neuropathy with probable pre-existing vascular compromise of the optic nerve.

Due to poor control of systemic symptoms of thyrotoxicosis the patient remains under the supervision of physicians and has been referred for radioactive iodine therapy.

\section{DISCUSSION}

Surgical decompression of the orbit is indicated in those patients with dysthyroid eye disease who have symptoms and signs of compressive optic neuropathy and who are refractory to or intolerant of systemic corticosteroid therapy. This surgery aims to reduce the intraorbital pressure by allowing the prolapse of the congested orbital contents into extraorbital space. The paranasal air sinuses (maxillary, ethmoidal and sphenoidal air sinuses) are suitably positioned to provide this space. Many surgical approaches have been used. A combination of inferior and medial orbital wall removal (antralethmoidal) with longitudinal incision of the periorbita was described by Walsh and Ogura ${ }^{7}$ and is now frequently used. The approach to the orbital floor may be trans-conjunctivally or trans-antrally; the medial wall is approached by either a skin incision or trans-conjunctivally. However, this results in a surgical scar and possible antral pain. Many other complications have been reported with varying frequencies, including extraocular muscle imbalance with diplopia, sinusitis, lower lid entropion and infraorbital nerve paraesthesia with lip numbness. ${ }^{8,9}$

In normal orbital anatomy, the optic foramen is in the lesser wing of the sphenoid. The posterior ethmoidal and sphenoidal sinuses form the medial 
wall of the optic canal and the posterior extremity of the medial orbital wall is formed by the sphenoid bone. In our 3 patients the anterior limit of the sphenoidal sinus was more anterior than usually seen. We recognise this as a variant of the normal orbital anatomy. Decompression into the sphenoidal sinus should be advantageous in such patients who have optic nerve compression. However, access to the posteriorly placed sphenoidal sinus is limited and may be hazardous with an orbital approach, so that the potential of this space is not realised.

The advent of endoscopes has significantly improved visualisation and access to the paranasal sinuses. Application of this technology to ophthalmic practice for orbital decompression in dysthyroid eye disease has given encouraging results. ${ }^{10-12}$ An endonasal endoscopic approach for inferomedial decompression in compressive optic neuropathy has been described ${ }^{13,14}$ and has recently been shown to give good protective capacity to avoid recurrence of compressive optic neuropathy. ${ }^{15}$

In the 3 cases reported, the initial improvement in visual acuity following standard two-wall surgical decompression was not maintained, presumably due to progressive infiltration of the orbital tissue associated with active thyroid cye disease. We believe that the addition of sphenoidal decompression using the endoscopic technique improved the chance of a favourable visual outcome. Two patients (cases 1 and 2) had improved visual function. In the patient (case 3) who did not improve it was felt that chronic vascular compromise due to long-standing compression of the optic nerve reduced the visual potential of the eyes. It is difficult to say with certainty whether earlier more complete surgical decompression would have increased the chance of visual recovery.

No post-operative surgical complications occurred. One patient (case 3) had persistence of a previous motility problem. This was treated with squint surgery with a good result. The onset or progression of diplopia may not reflect a complication of surgery but may be the sequela of a good decompression, since new-onset diplopia is more common in patients undergoing more extensive decompressions. ${ }^{16}$

Careful review of CT scans is essential in the preoperative assessment of patients with dysthyroid eye disease. We emphasise the importance of recognising anatomical variations, in particular anterior placement of the sphenoidal air cells. We suggest that an endoscopic approach be considered in these patients if surgical decompression is required. This technique may allow for more complete optic nerve decompression. However, the risk of complications may be high for surgeons without considerable skill and experience. ${ }^{17.18}$ We encourage ophthalmic and oto- laryngological surgeons to collaborate and share techniques and expertise that will improve the overall management of patients with dysthyroid eye disease and compressive optic neuropathy.

Key words: Optic nerve compression, Dysthyroid eye disease, Sphenoidal sinus, Endoscopic decompression, Computed tomographic scan.

\section{REFERENCES}

1. Neigel JM, Rootman J, Belkin RI, et al. Dysthyroid optic neuropathy: the crowded orbital apex syndrome. Ophthalmology 1988;95:1515-21.

2. Char DH. Thyroid eye disease, 1st ed. Baltimore: Williams and Wilkins, 1985.

3. Trobe JD. Optic nerve involvement in dysthyroidism. Ophthalmology 1981;88:488-92.

4. Anderson RL, Linberg JV. Transorbital approach to decompression in Graves disease. Arch Ophthalmol 1981;99:120-4.

5. Rootman J. Graves' orbitopathy. In: Diseases of the orbit. New York: JB Lippincott, 1988:265.

6. Fujii K, Chambers SM, Rhoton AL. Neurovascular relationships of the sphenoid sinus: a microsurgical study. J Neurosurg 1979;5():31-9.

7. Walsh TE, Ogura JH. Transnasal orbital decompression for malignant exophthalmos. Laryngoscope 1957; 67:544-9.

8. Fatourechi V, Garrity JA, Bartley GB, Bergstralh EJ, DeSanto LW, Gorman CA. Graves' ophthalmopathy: results of transantral orbital decompression performed primarily for cosmetic indications. Ophthalmology 1994;101:938-42.

9. Garrity JA, Fatourechi V, Bergstrahl EJ, Bartley GB, Beatty CW, DeSanto LW, Gorman CA. Results of transantral orbital decompression in 428 patients with severe Graves' ophthalmopathy. Am J Ophthalmol 1993;116:533-47.

10. Warren JD, Spector JG, Burde R. Long term follow up and recent observations on 305 cases of orbital decompression for dysthyroid orbitopathy. Laryngoscope 1989;99:35-40.

11. Desanto LW. The total rehabilitation of Graves' ophthalmopathy. Laryngoscope 1986;90:1652-78.

12. Khan JA, Wagner DV, Tiojanco JK, Hoover LA. Combined transconjunctival and external approach for endoscopic orbital apex decompression in Graves' disease. Laryngoscope 1995;105:203-6.

13. Kennedy DW, Goodstein ML, Miller NR, Zinreich SJ. Endoscopic transnasal orbital decompression. Arch Otolaryngol Head Neck Surg 1990;116:275-82.

14. Mann W, Kahaly G, Lieb W, Rothoff T, Springhorn S. Orbital decompression for endocrine ophthalmopathy: the endonasal approach. Dev Ophthalmol 1993;25: $142-50$.

15. Neugebauer A, Nishino K, Neugebauer P, Konen W, Michel O. Effects of bilateral orbital decompression by an endoscopic endonasal approach in dysthyroid orbitopathy. Br J Ophthalmol 1996;80:58-62.

16. McCord CD Jr. Current trends in orbital decompression. Ophthalmology 1985;92:21-33.

17. Buus RB, Tse DT, Farris BK. Ophthalmic complications of sinus surgery. Ophthalmology 1990;97:612-8.

18. Stankiewicz JA. Complications in endoscopic intranasal ethmoidectomy: an update. Laryngoscope 1989;99: 686-90. 